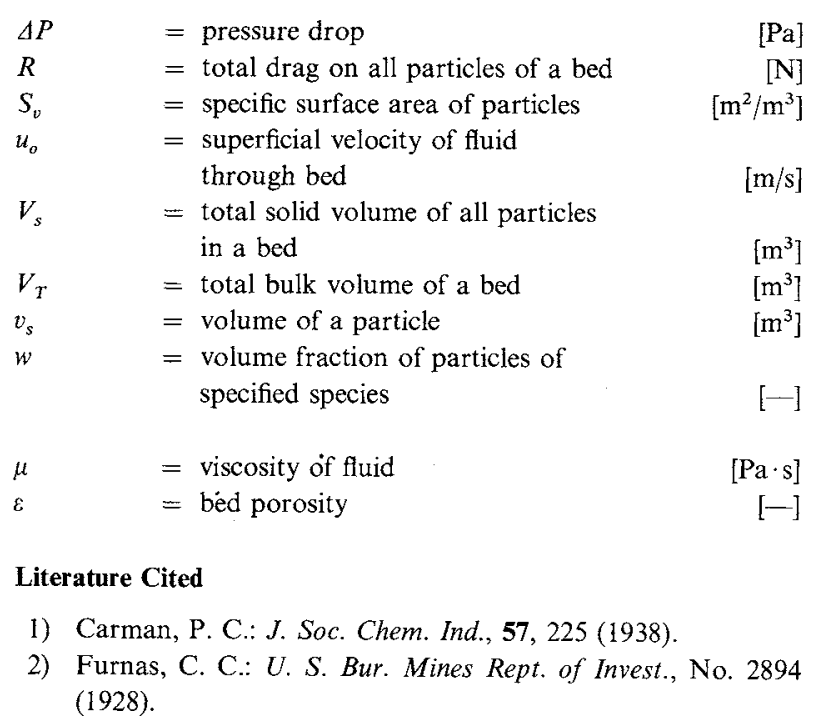

3) Garside, J. and M. R. Al-Dibouni: Ind. Eng. Chem., Process Des. Dev., 16, 206 (1977).

4) Kagaku Kogaku Kyokai-Hen: "Kagaku Kogaku Binran," p. 170, p. 976, p. 1141, Maruzen (1978).

5) Kagaku Kogaku Kyokai-Hen: "Kagaku Kogaku Binran," p. 1061, Maruzen (1978).

6) Moritomi, H., T. Iwase and T. Chiba: Chem. Eng. Sci, 37, 1751 (1982).

7) Moritomi, H., Y. Yamagishi and T. Chiba: Chem. Eng. Sci., 41, 297 (1986).

8) Patwardhan, V. S. and C. Tien: Chem. Eng. Sci., 40, 1052 (1985).

9) Ridgway, K. and K. J. Tarbuck: Chem. Process Eng., 49, 103 (1968).

10) Senoo, M.: "Kagaku Netsurikigaku 1" (translation into Japanese), p. 1-7, Misuzu Shobo (1966).

11) Westman, A. E. R. and H. R. Hugill: J. Am. Ceram. Soc., 13, 767 (1930).

\title{
NUMERICAL ANALYSIS OF THREE DIMENSIONAL VELOCITY PROFILE OF HIGHLY VISCOUS NEWTONIAN FLUID IN AN AGITATED VESSEL WITH PADDLE IMPELLER
}

\author{
SETSURO HIRAOKA, IKUHO YAMADA, TSUTOMU ARAGAKI \\ HIROYUKI NISHIKI, AKIO SATO AND TAKASHI TAKAGI \\ Department of Applied Chemistry, Nagoya Institute of Technology, Nagoya 466
}

\begin{abstract}
Key Words: Agitated Vessel, Paddle Impeller, Velocity Profile, Power Input, Discharge Flow, Numerical Analysis

A numerical algorithm for three-dimensional laminar flow in an agitated vessel was established by using the vector potential and the vorticity. This algorithm was applied to the numerical analysis of the velocity profile of a highly viscous Newtonian fluid in a closed cylindrical vessel with paddle impeller, because of the simplicity of the boundary conditions. The governing equations were solved by using the S.O.R. method. The calculated velocity profile agreed well with that observed by Yamamoto in the region between impeller and vessel bottom, where the bound ary condition in the numerical analysis almost satisfied his experimental conditions. As the flow pattern near the impeller was scarcely affected by the nonexistence of a free liquid surface, the numerical results for power input and discharge flow rate coincided well with those observed by Nagata and Yamamoto.
\end{abstract}

\section{Introduction}

In chemical and allied industries the mixing of highly viscous fluid is frequently carried out in an agitated vessel. Mixing performance factors, such as power input, mixing time and heat transfer rate, are closely related to three-dimensional flow in the vessel, so flow patterns have been measured intensively by many investigators during the last three decades.

Nagata $^{14)}$ and Yamamoto ${ }^{19)}$ measured three-

Received April 28, 1987. Correspondence concerning this article should be addressed to $\mathrm{S}$. Hiraoka. dimensional velocity distributions by using streak line photographs of submerged small particles in viscous fluid, and obtained the relationship between discharge flow rate and power input. Peter and Smith ${ }^{17)}$ measured the stream line by using a cine-record of the movement of small suspended beads, and discussed the relation of mixing time to geometrical parameters. Murakami et al. ${ }^{13)}$ also measured three-dimensional flow patterns in typical agitated vessels from the streak-line photographs of polystyrene beads tracer, and obtained the distributions of energy dissipation and shear rate. 
Recently, numerical analysis of the velocity profile of highly viscous fluid in mixing devices has been conducted by many investigators. ${ }^{1,6,7,10,15,18)}$ As merits in numerical analysis, the resultant flow pattern gives rise to (i) theoretical verification of empirical correlation equations in mixing characteristics, (ii) derivation of accurate local values of flow characteristics, such as shear rate and dissipation function, and (iii) finding of characteristic quantities, such as length and velocity, which specify the flow and mixing performances. In most papers, the flow pattern was projected onto a two-dimensional (2-D) domain, the $r-\theta$ or $r-z$ domain in cylindrical coordinates, to simplify the numerical procedure. The resultant 2-D flow pattern derived power input, shear stress distribution, etc., and the validity of the concept of the average shear rate proposed by Metzner-Otto ${ }^{12)}$ was confirmed. However, the discharge flow rate, which specifies mixing characteristics, cannot be obtained from a 2-D flow analysis, because the discharge flow is secondary flow induced from the primary rotational flow. In other words, the secondary flow characteristics can only be obtained from a three-dimensional (3D) flow analysis.

A numerical analysis of turbulent flow, which is of primary inportance in engineering problems, was conducted in a few studies, ${ }^{4,16)}$ but the proposed results of flow pattern and flow characteristics were not satisfactory because of the complexity of the governing equations and boundary conditions.

In this paper, as the first step in establishing a numerical algorithm for 3-D flow analysis, we analyse the 3-D laminar flow pattern in a closed cylindrical vessel with paddle impeller, because this involves the simplest boundary conditions for the vessel wall.

\section{Governing Equations}

For a closed cylindrical vessel with paddle impeller, the vorticity equation at steady state can be expressed in the following form at the moving frame with constant angular velocity $\Omega$.

$$
\operatorname{Re} \vec{V} \times\left[\left(\vec{\zeta}_{\text {rot }}+2 \vec{\Omega}^{*}\right) \times \vec{v}_{\text {rot }}\right]=\vec{V}^{2} \vec{\zeta}_{\text {rot }}
$$

The vorticity vector $\vec{\zeta}_{\text {rot }}$ and the velocity vector $\vec{v}_{\text {rot }}$, respectively, are expressed by the vector potential $\vec{\psi}$, as follows (see Appendix A).

$$
\begin{aligned}
& \vec{\zeta}_{\text {rot }}=-\left[\vec{V}^{2} \vec{\psi}\right] \\
& \vec{v}_{\text {rot }}=[\vec{V} \times \vec{\psi}]
\end{aligned}
$$

In Eqs. (1) to (3), the subscript "rot" means the rotating frame, and all variables are nondimensionalized with the vessel radius, $R$, and the angular velocity of the impeller, $\Omega$, as the characteristic quantities. Then the apparent rotational velocity of the vessel wall, $V(\equiv R \Omega)$, observed on the rotating

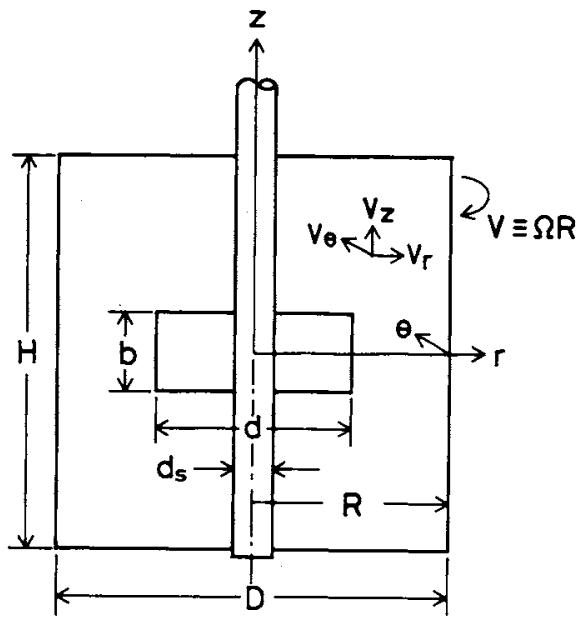

Fig. 1. Dimensions of closed cylindrical vessel with paddle impeller

frame with $\Omega$ becomes the characteristic velocity. The Reynolds number, $R e$, is also defined as $R V / v$ (see Fig. 1).

The boundary conditions for vector potential components on a solid wall at rest are specified in the same manner as by Hirasaki and Hellums, ${ }^{77}$ as follows:

$$
\frac{\partial \psi_{n}}{\partial n}=0, \quad \psi_{t}=0
$$

where $n$ and $t$ are normal and tangential components or coordinates to the solid surface at rest, respectively. Even though there is no precise expression for boundary conditions at a moving solid surface, they are assumed to be

$$
\frac{\partial \psi_{n}}{\partial n}=f(t), \quad \psi_{t}=g(t)
$$

where the functional forms of $f(t)$ and $g(t)$ are selected to satisfy both mass and force balances.

Under the cylindrical coordinates rotating with constant angular velocity $\Omega$ around $z$ axis, as shown in Fig. 1, the vorticity vector $\vec{\zeta}_{\text {rot }}$, the vector potential $\vec{\psi}$ and the velocity vector $\vec{v}_{\text {rot }}$, respectively, can be expressed by the vector components, as follows:

$$
\left.\begin{array}{l}
\vec{\zeta}_{\text {rot }}=\left[\zeta_{r}, \zeta_{\theta}, \zeta_{z}\right] \\
\vec{\psi}=\left[\psi_{r}, \psi_{\theta}, \psi_{z}\right] \\
\vec{v}_{\text {rot }}=\left[v_{r}, v_{\theta}, v_{z}\right]
\end{array}\right\}
$$

By using the vector components in Eq. (6), Eqs. (1), (2) and (3) are rearranged to

$$
\begin{aligned}
\operatorname{Re}\left[\left\{v_{r} \frac{\partial}{\partial r}+\frac{v_{\theta}}{r} \frac{\partial}{\partial \theta}+v_{z} \frac{\partial}{\partial z}\right\} \zeta_{i}\right. \\
\left.-\left\{\zeta_{r} \frac{\partial}{\partial r}+\frac{\zeta_{\theta}}{r} \frac{\partial}{\partial \theta}+\zeta_{z} \frac{\partial}{\partial z}\right\} v_{i}+a_{i}\right] \\
=\left\{\frac{\partial^{2}}{\partial r^{2}}+\frac{1}{r} \frac{\partial}{\partial r}+\frac{1}{r^{2}} \frac{\partial^{2}}{\partial \theta^{2}}+\frac{\partial^{2}}{\partial z^{2}}\right\}_{i}+b_{i} \quad[i=r, \theta, z]
\end{aligned}
$$


Table 1. Terms in Eqs. (7) and (8)

\begin{tabular}{cccc}
\hline$i$ & $a_{i}$ & $b_{i}$ & $c_{i}$ \\
\hline$r$ & 0 & $-\frac{2}{r^{2}} \frac{\partial \zeta_{\theta}}{\partial \theta}-\frac{\zeta_{r}}{r^{2}}$ & $-\frac{2}{r^{2}} \frac{\partial \psi_{\theta}}{\partial \theta}-\frac{\psi_{r}}{r^{2}}$ \\
\hline$\theta$ & $\frac{v_{\theta} \zeta_{r}-v_{r} \zeta_{\theta}}{r}$ & $\frac{2}{r^{2}} \frac{\partial \zeta_{r}}{\partial \theta}-\frac{\zeta_{\theta}}{r^{2}}$ & $\frac{2}{r^{2}} \frac{\partial \psi_{r}}{\partial \theta}-\frac{\psi_{\theta}}{r^{2}}$ \\
\hline$z$ & 0 & 0 & 0 \\
\hline
\end{tabular}

$$
\begin{array}{r}
\zeta_{i}=-\left\{\frac{\partial^{2}}{\partial r^{2}}+\frac{1}{r} \frac{\partial}{\partial r}+\frac{1}{r^{2}} \frac{\partial^{2}}{\partial \theta^{2}}+\frac{\partial^{2}}{\partial z^{2}}\right\} \psi_{i}+c_{i} \\
{[i=r, \theta, z]}
\end{array}
$$

and

$$
\left.\begin{array}{l}
v_{r}=\frac{1}{r} \frac{\partial \psi_{z}}{\partial \theta}-\frac{\partial \psi_{\theta}}{\partial z} \\
v_{\theta}=\frac{\partial \psi_{r}}{\partial z}-\frac{\partial \psi_{z}}{\partial r} \\
v_{z}=\frac{1}{r} \frac{\partial r \psi_{\theta}}{\partial r}-\frac{1}{r} \frac{\partial \psi_{r}}{\partial \theta}
\end{array}\right\}
$$

respectively, were $a_{i}, b_{i}$ and $c_{i}$ in Eqs. (7) and (8) are listed in Table 1.

The solenoidal condition for the vector potential, Eq. (A-5) in Appendix A, is expressed as

$$
\frac{1}{r} \frac{\partial r \psi_{r}}{\partial r}+\frac{1}{r} \frac{\partial \psi_{\theta}}{\partial \theta}+\frac{\partial \psi_{z}}{\partial z}=0
$$

The boundary conditions for each vector component are derived from the relations of Eqs. (4) and (5), and are listed in Table 2, where the vessel height $H$ is equal to the vessel diameter $D$ and the shaft diameter $d_{s}$ to one-tenth of the vessel diameter, for simplicity. The conditions for a moving boundary, VI) and VII) in Table 2, contain two unknown parameters, $C_{1}$ and $C_{2}$, that determine the functional form in Eq. (5) (see Appendix B). These unknown parameters are specified to maintain the conservation of angular momentum in both $r$ - and $z$-directions, i.e., $r$-direction

$$
\begin{gathered}
\int_{0}^{2 \pi}\left[\int_{-1}^{1}\left[r \zeta_{z}\right]_{d / D}^{1} d z-\int_{-1}^{1} \int_{d / D}^{1} \zeta_{z} d r d z-\int_{d / D}^{1}\left[r \zeta_{r}\right]_{-1}^{1} d r\right. \\
\left.-\operatorname{Re} \int_{-1}^{1} \int_{d / D}^{1}\left\{v_{r}\left(\zeta_{z}+2 \Omega^{*}\right)-v_{z} \zeta_{r}\right\} r d r d z\right] d \theta=0
\end{gathered}
$$

$z$-direction

$$
\int_{0}^{2 \pi}\left[\int_{b / H}^{1}\left[r \zeta_{z}\right]_{d s / D}^{1} d z-\int_{b / H}^{1} \int_{d / D}^{1} \zeta_{z} d r d z-\int_{d s / D}^{1}\left[r \zeta_{r}\right]_{b / H}^{1} d r-R e \int_{b / H}^{1} \int_{d s / D}^{1}\left\{v_{r}\left(\zeta_{z}+2 \Omega^{*}\right)-v_{z} \zeta_{r}\right\} r d r d z\right] d \theta=0
$$

Table 2. Boundary conditions

(A) Fixed boundary $\left(v_{r}=v_{\theta}=v_{z}=0\right)$

(I) Shaft ( $r_{s}=0.1, z-\theta$ surface)

$$
\begin{aligned}
& \partial r \psi_{r} / \partial r=0, \quad \psi_{\theta}=0, \quad \psi_{z}=0 \\
& \zeta_{r}=0, \quad \zeta_{\theta}=-\left\{\frac{\partial}{\partial r}\left(\frac{1}{r} \frac{\partial r \psi_{\theta}}{\partial r}\right)+\frac{2}{r^{2}} \frac{\partial \psi_{r}}{\partial \theta}\right\} \\
& \zeta_{z}=-\frac{1}{r} \frac{\partial}{\partial r}\left(r \frac{\partial \psi_{z}}{\partial r}\right)
\end{aligned}
$$

(II) Impeller $(r-z$ surface)

$$
\begin{aligned}
& \psi_{r}=0, \quad \partial \psi_{\theta} / \partial \theta=0, \quad \psi_{z}=0 \\
& \zeta_{r}=-\frac{1}{r^{2}} \frac{\partial^{2} \psi_{r}}{\partial \theta^{2}}, \quad \zeta_{\theta}=0, \quad \zeta_{z}=-\frac{1}{r^{2}} \frac{\partial^{2} \psi_{z}}{\partial \theta^{2}}
\end{aligned}
$$

(III) Impeller corner ( $r-\theta$ surface)

$$
\begin{array}{ll}
\psi_{r}=0, \quad \partial \psi_{\theta} / \partial \theta=0, & \psi_{z}=0 \\
\zeta_{r}=-\left\{\frac{1}{r^{2}} \frac{\partial^{2} \psi_{r}}{\partial \theta^{2}}+\frac{\partial^{2} \psi_{r}}{\partial z^{2}}\right\}, & \zeta_{\theta}=0, \quad \zeta_{z}=0
\end{array}
$$

(IV) Impeller corner ( $z-\theta$ surface)

$$
\begin{aligned}
& \psi_{r}=0, \quad \partial \psi_{\theta} / \partial \theta=0, \quad \psi_{z}=0 \\
& \zeta_{r}=0, \quad \zeta_{\theta}=0, \\
& \zeta_{z}=-\left\{\frac{1}{r} \frac{\partial}{\partial r}\left(r \frac{\partial \psi_{z}}{\partial r}\right)+\frac{1}{r^{2}} \frac{\partial^{2} \psi_{z}}{\partial \theta^{2}}\right\}
\end{aligned}
$$

(V) Impeller corner tip

$$
\begin{aligned}
& \psi_{r}=0, \quad \partial \psi_{\theta} / \partial \theta=0, \quad \psi_{z}=0 \\
& \zeta_{r}=-\frac{1}{2}\left\{\frac{1}{r^{2}} \frac{\partial^{2} \psi_{r}}{\partial \theta^{2}}+\frac{\partial^{2} \psi_{r}}{\partial z^{2}}\right\}, \quad \zeta_{\theta}=0, \\
& \zeta_{z}=-\frac{1}{2}\left\{\frac{1}{r} \frac{\partial}{\partial r}\left(r \frac{\partial \psi_{z}}{\partial r}\right)+\frac{1}{r^{2}} \frac{\partial^{2} \psi_{z}}{\partial \theta^{2}}\right\}
\end{aligned}
$$

(B) Moving boundary $\left(v_{\theta}=-\Omega^{*} r, \quad v_{r}=v_{z}=0\right)$

(VI) Side wall of vessel $(z-\theta$ surface $)$

$$
\begin{aligned}
& \frac{1}{r} \frac{\partial r \psi_{r}}{\partial r}=C_{1} z, \quad \psi_{\theta}=0, \quad \psi_{z}=-\frac{1}{2} C_{1} z^{2}+C_{2} \\
& \zeta_{r}=0, \quad \zeta_{\theta}=-\left\{\frac{\partial}{\partial r}\left(\frac{1}{r} \frac{\partial r \psi_{\theta}}{\partial r}\right)+\frac{2}{r} \frac{\partial^{2} \psi_{r}}{\partial \theta^{2}}\right\}, \\
& \zeta_{z}=-\frac{1}{r} \frac{\partial}{\partial r}\left(r \frac{\partial \psi_{z}}{\partial r}\right)
\end{aligned}
$$

(VII) Bottom $(z=-1)$ and top $(z=1)$ walls of vessel $(r-\theta$ surface $)$

$$
\begin{aligned}
& \psi_{r}= \pm \frac{1}{2} C_{1}\left(r-\frac{r_{s}^{2}}{r}\right), \quad \psi_{\theta}=0, \quad \frac{\partial \psi_{z}}{\partial z}=\mp C_{1} \\
& \zeta_{r}=-\frac{\partial^{2} \psi_{r}}{\partial z^{2}}, \quad \zeta_{\theta}=-\frac{\partial^{2} \psi_{\theta}}{\partial z^{2}}, \quad \zeta_{z}=2 \Omega^{*}
\end{aligned}
$$




\section{Numerical Procedure}

In the numerical analysis, the flow field is divided into $N_{r} \times N_{\theta} \times N_{z}$ sub-fields as shown in Fig. 2, and Eqs. (7) and (8) are transformed into finite-difference equations at a nodal point $(i, j, k)$ by using sevenpoint-difference form. The boundary conditions in Table 2 are also transformed into finite-difference forms. The successive over-relaxation (S.O.R.) method is applied to solve these difference equations. The numerical algorithm is almost the same as in 2-D flow analysis ${ }^{6}{ }^{6}$ as shown in Fig. 3.

The computational procedure is as follows:

Step 1: $\quad C_{1}$ and $C_{2}$ are assumed.

Step 2: $\psi$ and $\zeta$ are computed by S.O.R. method.

Step 3: Check whether Eqs. (11) and (12) hold simultaneously under convergence criteria or not.

Step 4: If not, reassume either $C_{1}$ and $C_{2}$ and do steps 2 and 3 .

Step 5: If so, real solutions of $\psi$ and $\zeta$ are obtained.

Step 6: Velocity profiles are calculated from $\psi$.

In this study the number of divisions $N_{r} \times N_{\theta} \times N_{z}$ is $18 \times 20 \times 40$. Total job memory is about $1800 \mathrm{~KB}$ and one iteration time is $1.8 \mathrm{~s}$ by a HITAC-M260D computer. The convergence criterion for Eqs. (11) and (12) is set at $10^{-3}$; then the number of iterations for given $C_{1}$ and $C_{2}$ is about 250 . The Reynolds number, $R V / v$, is varied in a range less than $10^{2}$, in which the flow is supposed to be laminar.

\section{Results and Discussion}

\subsection{Velocity profile}

The average velocity profile on the $r-z$ plane for a paddle of $d / D=0.5, n_{p}=8$ and the operating conditions of $R e_{d}=14$ is shown in Fig. 4, where the secondary circulation flow has two vortex centers near the upper and lower tips of the impeller blades. The velocity profile on the $r-\theta$ plane near the bottom wall is shown in Fig. 5 for the same impeller and the same Reynolds number as in Fig. 4, where the liquid flows inward with rotational flow. Distributions of the average real tangential velocity components $v_{\theta}^{\prime}$ and the average radial velocity component $v_{r}$ are shown in Figs. 6 and 7, respectively. The dotted line in these figures expresses the observed velocity distribution by Yamamoto. ${ }^{19)}$ The diameter of the agitated vessel with flat bottom used by Yamamoto, $D$, was $58.5 \mathrm{~cm}$, the liquid depth ratio $H / D=1.0$, the impeller diameter ratio $d / D=0.5$, the impeller width ratio $b / D=0.1$ and the shaft diameter ratio $d_{s} / D=0.03$. The impeller was fixed at the center of the vessel. The boundary conditions in the present analysis satisfy the experimental conditions of Yamamoto for the region between impeller and vessel bottom, except for the shaft diameter ratio. It is clear from these fig.

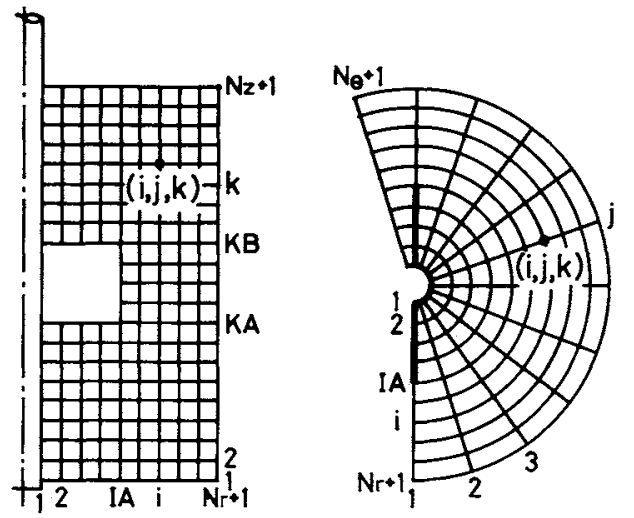

Fig. 2. Schematic diagram of division for difference approximation of governing equations

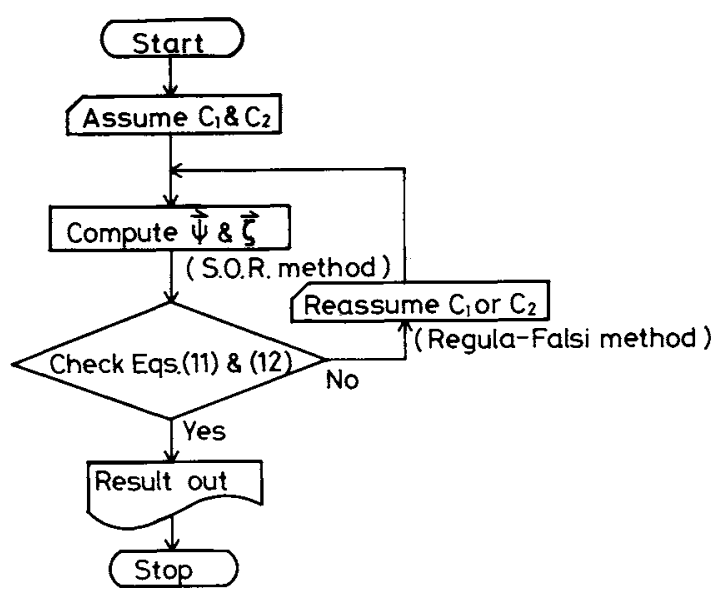

Fig. 3. Flow chart for numerical procedure

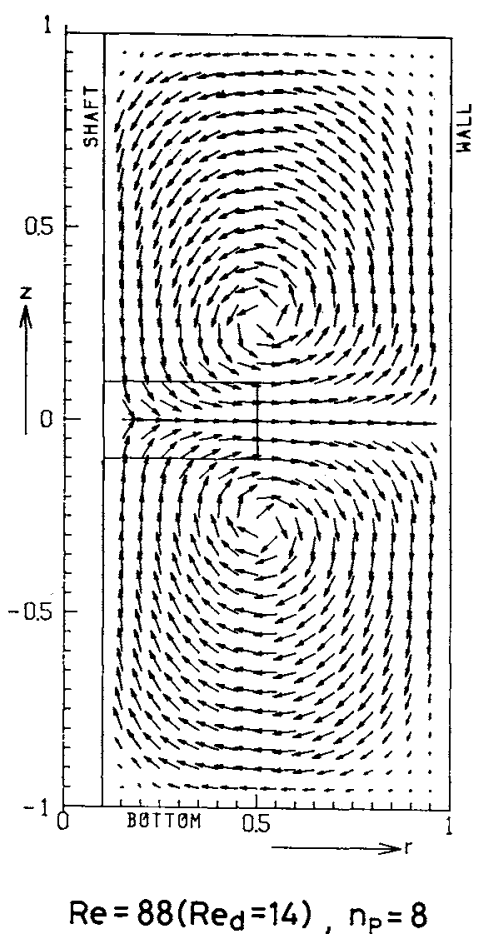

Fig. 4. Average velocity profile on $r-z$ plane 


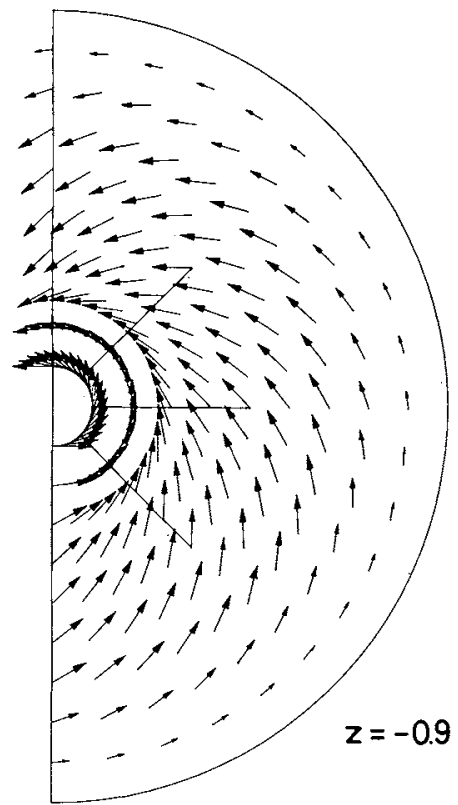

$\operatorname{Re}=88\left(\operatorname{Re}_{\mathrm{d}}=14\right), n_{\mathrm{p}}=8$

Fig. 5. Velocity profile on $r-\theta$ plane near bottom wall

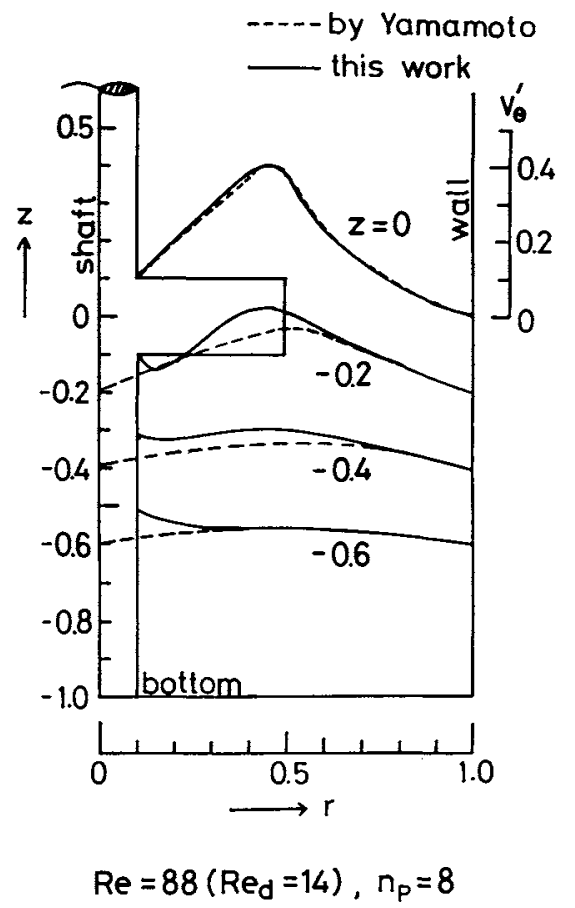

Fig. 6. Distribution of average real tangential velocity component

ures that the calculated and observed velocity profiles in this region are in very good agreement with each other except in the area near the shaft.

\subsection{Power input}

The dimensionless average shear stress on the side wall of the vessel, $\tau_{w}^{*}$, can be calculated from the vorticity distribution on the side wall as follows:

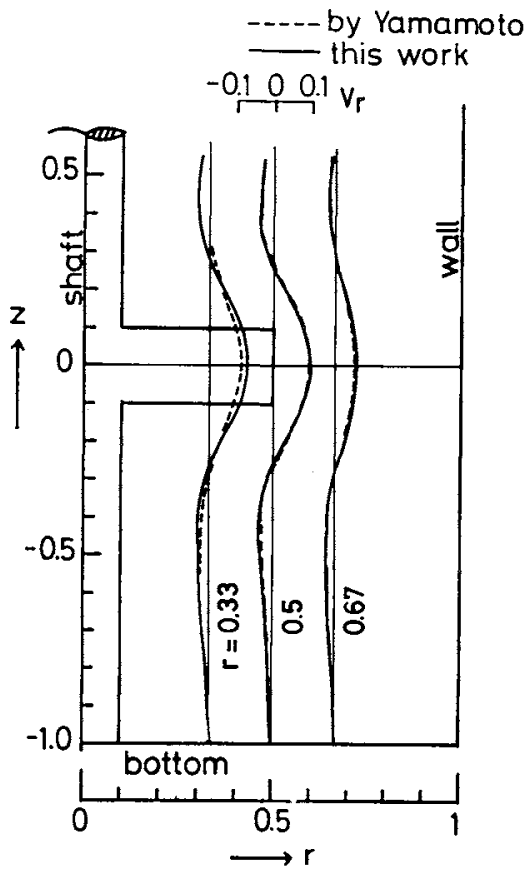

$$
\operatorname{Re}=88\left(\operatorname{Re}_{d}=14\right), n_{p}=8
$$

Fig. 7. Distribution of average radial velocity component at impeller tip

$$
\bar{\tau}_{w}^{*}=\frac{\bar{\tau}_{w}}{\mu(V / R)}=\frac{1}{4 \pi} \int_{0}^{2 \pi} \int_{-1}^{1}\left(\left.\zeta_{z}\right|_{r=1}-2\right) d z d \theta
$$

The ratio $\alpha$ of the total torque on top and bottom wall $T_{B}$ to that on side wall $T_{w}$ can be expressed as

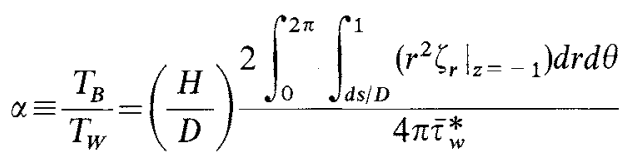

Then the power input $P$ can be obtained from the following relation: ${ }^{53}$

$$
P=\left(\frac{V}{R}\right)\left(T_{W}+T_{B}\right)=\left(\frac{V}{R}\right)(1+\alpha)\left(2 \pi R^{2} H \bar{\tau}_{w}\right)
$$

This equation can be rearranged to the following dimensionless form:

$$
N_{P} \cdot R e_{d}=\frac{P}{\mu N^{2} d^{3}}=2 \pi^{3}(1+\alpha)\left(\frac{D^{2} H}{d^{3}}\right) \bar{\tau}_{w}^{*}
$$

The product of power number and Reynolds number obtained from the numerical analysis of 3-D velocity profile via Eqs. (13) to (16) is plotted in Fig. 8 for $R e_{d}<1$, where the solid line expresses the empirical equation by Nagata ${ }^{14)}$

$$
N_{p} \cdot R_{d}=14+(b / D)\left\{670(d / D-0.6)^{2}+185\right\}
$$

in the range of $d / D=0.4-0.6$, and the dotted line expresses the semi-empirical equation by Hiraoka ${ }^{8)}$ 


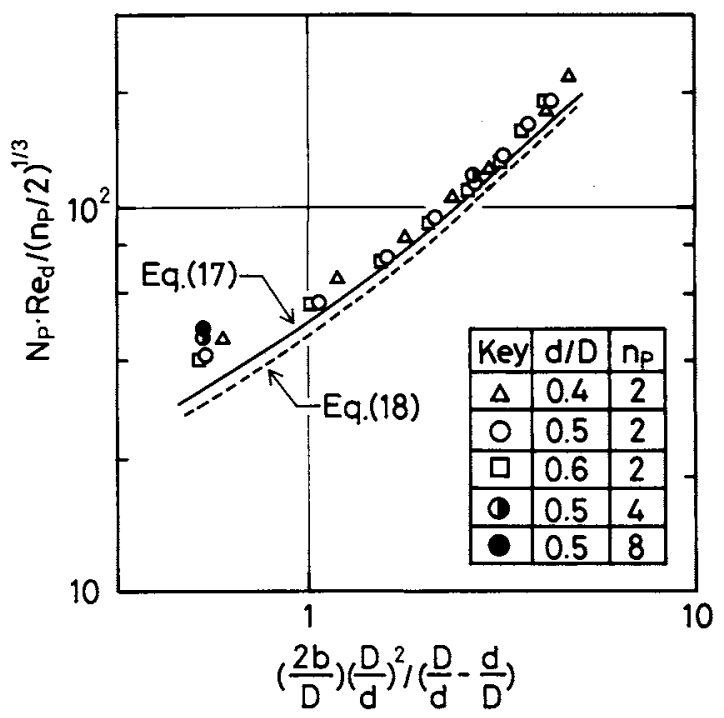

Fig. 8. Correlation of power input with impeller dimensions in small Reynolds number range of $R e_{d}<1$

$$
\frac{N_{P} \cdot R e_{d}}{\left(n_{p} / 2\right)^{1 / 3}}=13+34\left(\frac{1}{\eta}\right)\left(\frac{2 b}{D}\right) \frac{(D / d)^{2}}{(D / d-d / D)}
$$

for a wide range of $d / D$. This figure confirms that the calculated power input is very close to the observed one, Eqs. (17) or (18). This means that the power input is strongly affected by the flow pattern around the impeller.

The dependency of $N_{p} \cdot R e_{d}$ on $R e_{d}$ in 3-D flow analysis was very similar to that in 2-D flow analysis in the range of $R e_{d}<20$ for impellers of all sizes.

\subsection{Discharge flow rate}

The dimensionless ratio of power input to discharge flow rate is plotted against Reynolds number in Fig. 9, where discharge flow number $N_{q d}$ is defined as

$$
N_{q d}=\frac{Q_{d}}{N d^{3}}=\frac{\pi}{4}\left(\frac{D H}{d^{2}}\right) \int_{0}^{2 \pi} \int_{-b / H}^{b / H}\left(\left.v_{r}\right|_{r=d / D}\right) d z d \theta
$$

As shown in Fig. 9, the ratio $N_{p} / N_{q d}$ was almost independent of impeller size, and decreased rapidly with increase of $R e_{d}$. In other words, the discharge flow rate per unit power input increases rapidly with increasing Reynolds number. The observed ratio by Yamamoto $^{19)}$ is plotted in the same figure, where the calculated value agrees well with the observed one, despite of the nonexistence of a free liquid surface.

\section{Conclusion}

A numerical algorithm for three-dimensional laminar flow in a closed agitated vessel with paddle impeller was established by using the vector potential and the vorticity. The calculated velocity profile agreed well with that observed by Yamamoto in the region between impeller and vessel bottom, where the boundary conditions in the analysis almost satisfied

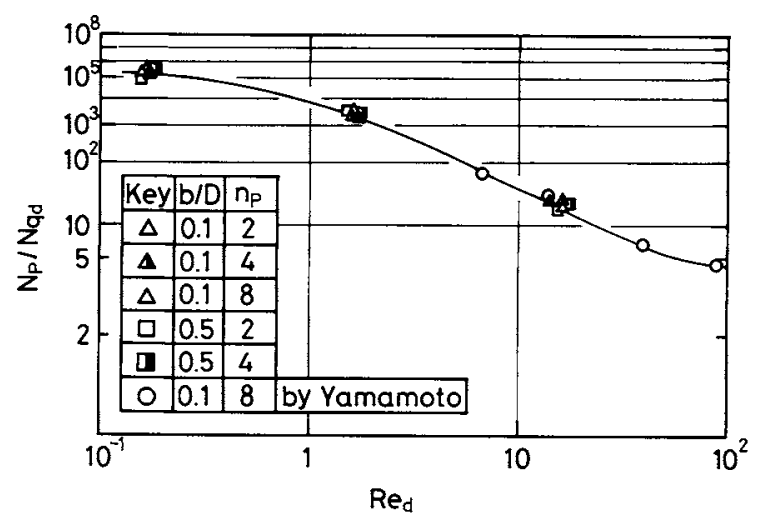

Fig. 9. Dimensionless ratio of power input to discharge flow rate

his experimental conditions. The numerical results of power input and discharge flow rate coincided well with the experimental results of Nagata and Yamamoto, because the flow pattern near the impeller was scarcely affected by the nonexistence of a free liquid surface.

Appendix A. Derivation of governing equations in rotating frame

In a cylindrical coordinate system rotating with constant angular velocity $\Omega$, the vorticity equation can be expressed as ${ }^{3)}$

$$
\left.\frac{\partial \vec{\zeta}_{\mathrm{rot}}}{\partial t}+\vec{V} \times\left[\vec{\zeta}_{\mathrm{rot}}+2 \vec{\Omega}\right) \times \vec{v}_{\mathrm{rot}}\right]=-v \vec{V} \times\left[\vec{V} \times \vec{\zeta}_{\mathrm{rot}}\right]
$$

where the subscript "rot" denotes a variable based on the rotating frame.

The definition of the vorticity $\vec{\zeta}_{\text {rot }}$ is

$$
\vec{\zeta}_{\text {rol }}=\left[\vec{V} \times \vec{v}_{\text {rot }}\right]
$$

The equation of continuity for incompressible fluid is

$$
\left(\vec{V} \cdot \vec{v}_{\mathrm{rol}}\right)=0
$$

For a viscous fluid, the velocity vector $\vec{v}_{\text {rot }}$ can be expressed only by the vector potential $\vec{\psi}$, as follows ${ }^{2)}$ :

$$
\vec{v}_{\text {rot }}=[\vec{\nabla} \times \vec{\psi}]
$$

The vector potential satisfies the solenoidal condition, i.e.

$$
(\vec{V} \cdot \vec{\psi})=0
$$

Substitution of Eq. (A-4) into Eq. (A-2) gives rise to

$$
\vec{\zeta}_{\text {rot }}=\vec{\nabla} \times[\vec{\nabla} \times \vec{\psi}]
$$

where the right-hand side is rewritten as

$$
\vec{\nabla} \times[\vec{V} \times \vec{\psi}]=-[\vec{\nabla}(\nabla \cdot \vec{\psi})]-\left[\vec{\nabla}^{2} \vec{\psi}\right]
$$

The first term on the right-hand side is zero, because of the solenoidal condition of Eq. (A-5). Then Eq. (A-6) is reduced to

$$
\vec{\zeta}_{\text {rat }}=-\left[\vec{\nabla}^{2} \vec{\psi}\right]
$$

The right-hand side of Eq. (A-1) is also rewritten, in the same manner as Eq. (A-7), as

$$
\begin{aligned}
\vec{\nabla} \times\left[\vec{V} \times \vec{\zeta}_{\text {rot }}\right] & =-\left[\vec{\nabla}\left(\vec{\nabla} \cdot \vec{\zeta}_{\text {rot }}\right)\right]-\left[\vec{\nabla}^{2} \vec{\zeta}_{\text {rot }}\right] \\
& =-\left[\vec{\nabla}^{2} \vec{\zeta}_{\text {rot }}\right]
\end{aligned}
$$

Then Eq. (A-1) is reduced to

$$
\frac{\partial \vec{\zeta}_{\mathrm{rot}}}{\partial t}+\vec{V} \times\left[\left(\vec{\zeta}_{\mathrm{rot}}+2 \vec{\Omega}\right) \times \vec{v}_{\mathrm{rot}}\right]=v\left[\vec{V}^{2} \vec{\zeta}_{\mathrm{rot}}\right]
$$




\section{Appendix B, Conditions at moving boundary ${ }^{11)}$}

As the vector potential $\vec{\psi}$ at the vessel wall moving at constant angular velocity $\Omega$ can be assumed to be uniform in $\theta$-direction, the derivative of the vector potential components with respect to $\theta$ is zero, and the component $\psi_{\theta}$ equals zero because of mass balance, i.e.

$$
\begin{aligned}
\frac{\partial \psi_{i}}{\partial \theta} & =0 \quad(i=r, \theta, z) \\
\psi_{\theta} & =0
\end{aligned}
$$

The derivative of the component $\psi_{r}$ with respect to $r$ on the side wall of the vessel can be expressed as the following equation, based on the relation of Eq. (5) in the text.

$$
\frac{1}{r} \frac{\partial r \psi_{r}}{\partial r}=F(z)
$$

By using both the solenoidal condition, Eq. (10) in the text, and the relation of Eq. (B-1), and by expressing the function $F(z)$ in the simplest polynominal form, Eq. (B-2) is reduced to

$$
\frac{1}{r} \frac{\partial r \psi_{r}}{\partial r}=-\frac{\partial \psi_{z}}{\partial z}=C_{0}+C_{1} z
$$

The integration of Eq. (B-3) with respect to $z$ gives rise to

$$
\psi_{z}=-C_{0} z-\frac{1}{2} C_{1} z^{2}+C_{2} \quad(\text { at } \quad r=1)
$$

on the side wall of the vessel. Equation (B-3) must hold at the intersection between the side wall and the top or bottom wall $(z= \pm 1$ and $r=1)$, i.e.

$$
\frac{\partial \psi_{z}}{\partial z}=-C_{0} \mp C_{1}
$$

From both the continuousness of function and the solenoidal condition of Eq. (A-5), the following equation must hold, in the same manner as in Eq. (B-3), on the top and bottom wall surfaces.

$$
\frac{\partial \psi_{z}}{\partial z}=-\frac{1}{r} \frac{\partial r \psi_{r}}{\partial r}=-C_{0} \mp C_{1}
$$

The integration of $\psi_{r}$ with respect to $r$ gives rise to

$$
\psi_{r}=\left(\frac{C_{0} \pm C_{1}}{2}\right) r+\frac{C_{3}}{r}
$$

on the top and bottom walls, respectively. From the continuousness of $\psi_{r}$ at the intersection between the shaft and the top or bottom wall, Eq. (B-6) can be reduced to

$$
\psi_{r}=\left(\frac{C_{0} \pm C_{1}}{2}\right)\left(r-\frac{r_{s}^{2}}{r}\right) \quad(\text { at } z= \pm 1)
$$

When the impeller is fixed at the center of the closed cylindrical vessel, as shown in Fig. 1, the flow pattern must be symmetrical with respect to the surface of $z=0$. Then, Eqs. (B-3), (B-4), (B-6) and (B-8), respectively, are reduced to

$$
\left.\begin{array}{l}
\frac{1}{r} \frac{\partial r \psi_{r}}{\partial r}=C_{1} z \\
\psi_{z}=-\frac{C_{1}}{2} z^{2}+C_{2}
\end{array}\right\} \text { at } r=1
$$

These equations are listed in Table 2 .

\section{Nomenclature}

$a_{i}, b_{i}, c_{i}=$ terms in Eqs. (7) and (8) $\quad[-]$

$b \quad=$ impeller width [m]

$C_{0}, C_{1}, C_{2}=$ constants in Eq. (B-4) [-]

$C_{3} \quad=$ constant in Eq. (B-6) $\quad[-]$

D $=$ vessel diameter $[\mathrm{m}]$

$\begin{array}{lll}d & =\text { impeller diameter } & {[\mathrm{m}]}\end{array}$

$\begin{array}{lll}d_{s} & =\text { shaft diameter } & {[\mathrm{m}]}\end{array}$

$H \quad=$ vessel height $[\mathrm{m}]$

$N \quad=$ rotational speed $\left[\mathbf{s}^{-1}\right]$

$N_{p} \quad=$ Power number $\left(=P / \rho N^{3} d^{5}\right)$

$N_{q d} \quad=$ discharge flow number $\left(=Q_{d} / N d^{3}\right) \quad[-]$

$N_{r}, N_{\theta}, N_{z} \quad=$ number of divisions $\quad[-]$

$n_{p} \quad=$ number of impeller blades $\quad[-]$

$P \quad$ power input $\quad\left[\mathrm{kg} \cdot \mathrm{m}^{2} \cdot \mathrm{s}^{-3}\right]$

$Q_{d} \quad=$ discharge flow rate $\left[\mathrm{m}^{3} \cdot \mathrm{s}^{-1}\right]$

$R \quad=$ vessel radius $(=D / 2) \quad[\mathrm{m}]$

Re $\quad=$ Reynolds number $(=R V / v) \quad[-]$

$R e_{d} \quad=$ impeller Reynolds number $\left(=N d^{2} / \nu\right)$

$r \quad=$ dimensionless radius $\quad[-]$

$r_{s} \quad=$ dimensionless shaft radius $\quad[-]$

$T_{B} \quad=$ total torque on top and bottom wall

$\left[\mathrm{kg} \cdot \mathrm{m}^{2} \cdot \mathrm{s}^{-2}\right]$

$T_{W} \quad=$ torque on side wall $\quad\left[\mathrm{kg} \cdot \mathrm{m}^{2} \cdot \mathrm{s}^{-2}\right]$

$t \quad=$ time $\quad[-]$

$V \quad=$ apparent rotational velocity of vessel wall $(=\Omega R)$

$\vec{v}, v_{i} \quad=$ dimensionless velocity vector and its component

$v_{\theta}^{\prime} \quad=$ dimensionless real tangential velocity component $\left(=v_{\theta}+\Omega^{*} r\right)$

$z \quad=$ dimensionless axial coordinate $\quad[-]$

$\alpha \quad=$ torque ratio of total torque on top and bottom wall to that on side wall

$\vec{\zeta}, \zeta_{i}=$ dimensionless vorticity vector and its component

$[-]$

$\eta \quad=$ correction factor in Eq. (18) $(=1+\exp [-10\{(D / d)-1\}])$

$\theta \quad=$ tangential coordinate $\quad[-]$

$\mu \quad=$ viscosity $\quad[\mathrm{Pa} \cdot \mathrm{s}]$

$v \quad=$ kinematic viscosity $\quad\left[\mathrm{m}^{2} \cdot \mathrm{s}^{-1}\right]$

$\rho \quad=$ density $\left[\mathrm{kg} \cdot \mathrm{m}^{-3}\right]$

$\bar{\tau}_{W}^{*} \quad=$ dimensionless average shear stress on side wall $\quad[-]$

$\begin{array}{lll}\vec{\psi}, \psi_{i} & =\text { vector potential and its component } & {[-]} \\ \Omega & =\text { angular velocity of impeller } & {\left[\mathrm{rad} \cdot \mathrm{s}^{-1}\right]}\end{array}$

$\begin{array}{ll}\Omega & =\text { angular velocity of impeller [rad } \\ \vec{\Omega}^{*}, \Omega^{*} & =\text { dimensionless angular velocity vector and }\end{array}$ its component in rotational frame $\quad[-]$

$\langle$ Subscripts〉

$$
\begin{array}{ll}
n & =\text { normal } \\
t & =\text { tangential } \\
\text { rot } & =\text { rotational frame }
\end{array}
$$

\section{Literature Cited}

1) Bertrand, J. and J. P. Couderc: Can. J. Chem. Eng., 60, 738 (1982).

2) Davis, G. V. and M. Wolfshtein: Lecture Note in Physics, 35, 125 (1974).

3) Greenspan, H. P.: "Theory of Rotating Fluid," Cambridge University Press (1969).

4) Harvey, P. S. and M. Greaves: Trans. IChemE, 60, 201 (1982).

5) Hiraoka, S. and R. Ito: J. Chem. Eng. Japan, 6, 464 (1973).

6) Hiraoka, S., I. Yamada and K. Mizoguchi: J. Chem. Eng. Japan, 11, 487 (1978). 
7) Hiraoka, S., I. Yamada and K. Mizoguchi: J. Chem. Eng. Japan, 12, 56 (1979).

8) Hiraoka, S., I. Yamada, A. Sato and K. Sato: Bull. Nagoya Inst. Tech., 33, 279 (1981).

9) Hirasaki, G. J. and D. J. Hellums: Q. Appl. Math., 28, 293 (1970).

10) Kuriyama, H. Inomata, K. Arai and S. Saito: AIChE J., 28, 3 (1982).

11) Lin, A., G. V. Davis and J. A. Reizes: Lecture Note in Physics, 218, $380(1985)$

12) Metzner, A. B. and E. Otto: AIChE J., 3, 3 (1957).

13) Murakami, Y., K. Fujimoto, T. Shimada, A. Yamada and K.
Asano: J. Chem. Eng. Japan, 5, 297 (1972).

14) Nagata, S.: "Mixing, Principles and Applications," Kodansha A Halsted Press Book (1975).

15) Ohta, M., M. Kuriyama, K. Arai and S. Saito: J. Chem. Eng. Japan, 18, 81 (1985).

16) Placek, J., L. L. Travlarides, G. W. Smith and I. Fort: AIChE J., 32, 1771 (1986).

17) Peter, D. C. and J. M. Smith: Trans. Inst. Chem. Engr., $\mathbf{4 5}$, T360 (1967).

18) Spragg, A. J. P., S. J. Maskell and M. A. Patrick: 5th European Conference on Mixing, p. 507 (1985).

19) Yamamoto, K.: Ph.D. Thesis, Kyoto Univ. (1961).

\title{
EXTRACTION EQUILIBRIUM OF Ce(III), Pr(III) AND Nd(III) WITH ACIDIC ORGANOPHOSPHORUS EXTRACTANTS
}

\author{
YASUSHIGE MORI, HITOSHI OHYA, HARUHIKO ONO \\ AND WATARU EGUCHI \\ Department of Chemical Engineering, Kyoto University, Kyoto 606
}

Key Words: Extraction Equilibrium, Rare Earth, Acidic Organophosphorus Extractants, EHPNA, Additional Complex

\begin{abstract}
Solvent extraction has recently attracted major interest for industrial-scale separation of Ianthanoids, alike ion exchange. It is well known that di(2-ethylhexyl)phosphoric acid (henceforth D2EHPA) is an effective extractant for the mutual separation of lanthanoids, because of its high separation factor. 2-Ethylhexylphosphonic acid mono2-ethylhexyl ester (henceforth EHPNA), which is similar in its chemical structure as D2EHPA, has a higher ability of back extraction at low acid concentration than D2EHPA.

In this paper, the extraction equilibrium of cerium, praseodymium and neodymium by EHPNA was investigated and was compared with that by D2EHPA.

It was found that the existence of two kinds of complexes should be considered in the region of high concentration of EHPNA, unlike the case of D2EHPA. The extraction of metals from the solution of mixed lanthanoids can be explained based on the extraction mechanism of individual elements.
\end{abstract}

\section{Introduction}

As lanthanoids have superior characteristics such as in their fluorescent, magnetic, optical and catalytic properties, demand for them is increasing. But the mutual separation and purification of lanthanoid elements is difficult because of the similarity in their chemical nature. Solvent extraction has recently attracted major interest for industrial-scale separation of lanthanoids, alike ion exchange.

It is well known that di(2-ethylhexyl)phosphoric acid (henceforth D2EHPA) is an effective extractant for the mutual separation of lanthanoids, because of its high separation factor. But D2EHPA has the disadvantage of difficulty in back extraction. 2-

Received April 30, 1987. Correspondence conuerning this article should be addressed to $Y$. Mori. H. Ohya is now with National Res. Inst. for Poll. and Resources, Tukuba. H. Ono is now with The Nomura Securities Co., Ltd., Tokyo.
Ethylhexylphosphonic acid mono-2-ethylhexyl ester (henceforth EHPNA) has a higher ability of back extraction at low acid concentration than D2EHPA.

In this study, the extraction equilibrium of cerium, praseodymium and neodymium by EHPNA was investigated and was compared with that by D2EHPA.

\section{Experimental}

\subsection{Reagents}

EHPNA and D2EHPA were purified by washing the commercial extractants PC88A and DP8R kindly supplied by Daihachi Chemical Industry Co., Ltd., Japan, with $6 \mathrm{kmol} / \mathrm{m}^{3}$ hydrochloric acid solution, and then with deionized water several times. Toluene of guaranteed grade was used as the diluent without further purification. The organic solutions were prepared by diluting a desired amount of extractant with toluene. 